TRANSACTIONS OF THE

AMERICAN MATHEMATICAL SOCIETY

Volume 354, Number 7, Pages 2725-2743

S 0002-9947(02)02958-6

Article electronically published on March 11, 2002

\title{
ENRIGHT'S COMPLETIONS AND INJECTIVELY COPRESENTED MODULES
}

\author{
STEFFEN KÖNIG AND VOLODYMYR MAZORCHUK
}

\begin{abstract}
Let $\mathfrak{A}$ be a finite-dimensional simple Lie algebra over the complex numbers. It is shown that a module is complete (or relatively complete) in the sense of Enright if and only if it is injectively copresented by certain injective modules in the BGG-category $\mathcal{O}$. Let $A$ be the finite-dimensional algebra associated to a block of $\mathcal{O}$. Then the corresponding block of the category of complete modules is equivalent to the category of $e A e$-modules for a suitable choice of the idempotent $e$. Using this equivalence, a very easy proof is given for Deodhar's theorem (also proved by Bouaziz) that completion functors satisfy braid relations. The algebra $e A e$ is left properly and standardly stratified. It satisfies a double centralizer property similar to Soergel's "combinatorial description" of $\mathcal{O}$. Its simple objects, their characters and their multiplicities in projective or standard objects are determined.
\end{abstract}

\section{INTRODUCTION}

Let $\mathfrak{A}$ be a finite-dimensional simple Lie algebra over the complex numbers. Fix a triangular decomposition, $\mathfrak{A}=\mathfrak{N}_{-} \oplus \mathfrak{H} \oplus \mathfrak{N}_{+}$. Then the BGG-category $\mathcal{O}$, which has been defined in [BGG], decomposes into a direct sum of indecomposable subcategories, called blocks. Each block is equivalent to the module category of a finite-dimensional associative algebra.

In his study of fundamental series representations in E], Enright associated with each simple root of $\mathfrak{A}$ an endofunctor on the category of $\mathfrak{A}$-modules, which sends a module $M$ into what is called the relative completion of $M$. Relative completions preserve $\mathcal{O}$, and on a certain subcategory of $\mathcal{O}$ they can be written as the composition of a localization functor with restriction and with taking a certain locally nilpotent part $[\mathrm{De}, \mathrm{M}$.

In this paper we show that both the relative completions and Enright's absolute completions (from now on just called completions), which are compositions of certain relative completions, have very natural interpretations in terms of the finitedimensional algebras associated with blocks of $\mathcal{O}$. In fact, up to the equivalences constructed by [BGG, $[$ ], the (relatively or absolutely) complete modules (i.e., the images of completion functors) are precisely the injectively copresented modules for certain choices of injective modules. Thus Enright's completion fits into the general framework established by Auslander [A].

Received by the editors July 11, 2000 and, in revised form, October 3, 2001.

2000 Mathematics Subject Classification. Primary 17B10, 16G10.

The first author was partially supported by the EC TMR network "Algebraic Lie Representations" grant no ERB FMRX-CT97-0100.

The second author was an Alexander von Humboldt fellow at Bielefeld University. 
In particular, each block of the above categories of (relatively or absolutely) complete modules is equivalent to the module category of an algebra $e A e$, where $A$ is a block of $\mathcal{O}$ and $e$ is an idempotent, the primitive summands of which are naturally indexed by cosets of the Weyl group. Thus, these subcategories carry abelian structures, which are, in fact, not obtained by restriction from the abelian structure of $\mathcal{O}$.

From the abstract framework it is clear that the category of injectively copresented modules (for some choice of injectives) is equivalent to the category of projectively presented modules (for the corresponding choice of projectives). Explicit versions of the last categories occur in [BG, II 5.9] in the context of projective functors. There it is shown that certain translation functors are equivalences between categories of Harish-Chandra modules and categories of projectively presented modules in $\mathcal{O}$.

There is even another equivalent version of these categories, namely, a parabolic generalization of the category $\mathcal{O}$, which has been introduced and studied in FKM1, FKM2, FKM3 by Lie theoretic methods. For example, the abelian structure mentioned before was discovered in [FKM3] as a quite exotic looking property, which gets a natural explanation in the present framework.

Altogether we get the following picture - it contains five categories, corresponding blocks of which are all equivalent (for suitable choices of the defining parameters):

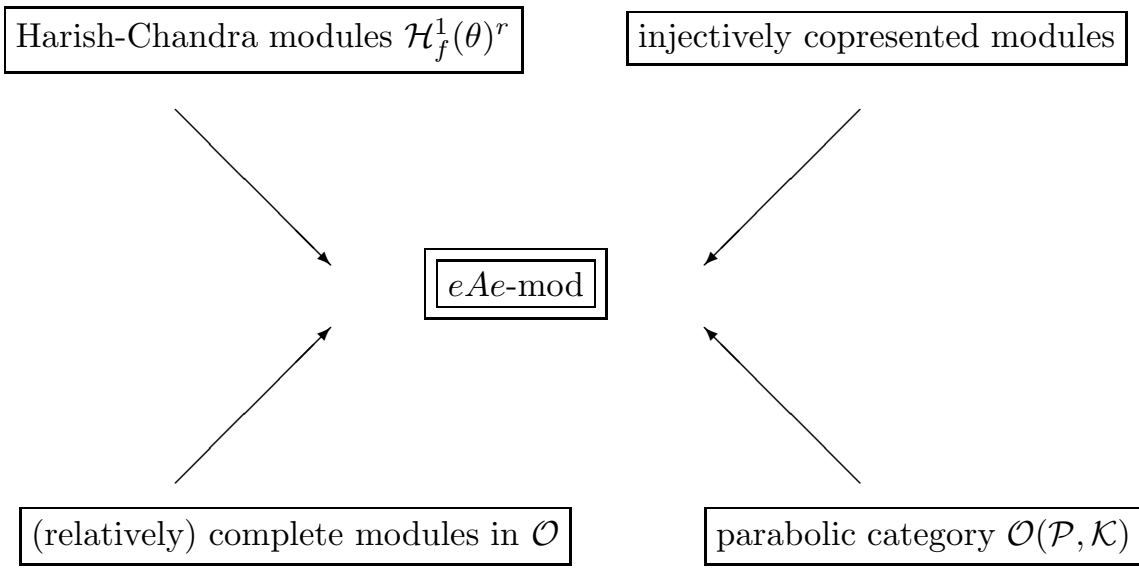

Having defined relative completions in [E], Enright posed the problem of showing that (on a certain subcategory) these relative completion functors satisfy the braid relations. This problem has been solved independently by Deodhar ([De] and by Bouaziz $([\mathrm{BO}])$. Later, Joseph $([\overline{J o}])$ extended the result to the whole category $\mathcal{O}$. It turns out that the subcategory considered by Deodhar is precisely the category of injectively cogenerated modules in our abstract setup, and this reformulation enables us to reprove the Bouaziz-Deodhar result in an easy way.

As a by-product of the proof we get that complete modules have a Verma flag. This yields a lower bound for the representation type of the category of modules having a Verma flag (and, of course, also for all of $\mathcal{O}$ ). See [BKM] for details of this application. 
The paper is naturally divided into two parts. The first part starts with Section 2] where we recall the abstract framework from Auslander's paper. Then, in Section 3 we define Enright's completion functors and show that the corresponding complete modules are precisely the injectively copresented modules when the injective module is chosen to be projective as well. More generally, in Section 4 we relate relatively complete modules to injectively copresented modules for certain choices of injectives. In Section 5 we reprove the validity of braid relations using our abstract approach. Section 6 recalls the equivalence proved in [BG] and then summarizes all the equivalences of categories we have obtained.

In the second part of the paper we consider the structure of the finite-dimensional algebras $e A e$ occurring above. In this way, we get several new results for the other four categories, but we also reprove in an easy way several assertions which were already known for some of these categories. In particular, we reprove and generalize results from [FKM3] on $\mathcal{S}$-subcategories in $\mathcal{O}$.

The basic structure of the algebra $A$ is that it is quasi-hereditary in the sense of Cline, Parshall and Scott CPS1. Verma modules play the role of standard modules in this structure. For a quasi-hereditary algebra $(A, \leq)$, in general it is known that certain centralizer subalgebras $e A e$ carry an induced quasi-hereditary structure (CPS1). However, this works only if the idempotent $e$ is associated to an ideal of the partially ordered set of weights. If $e$ is chosen in a different way, as it has to be in our situation, then a result of Dlab and Ringel ([DR] states that every finite-dimensional algebra can occur as $e A e$ for some quasi-hereditary algebra A.

Our aim in the second part of the paper is to show that for a rather different choice of $e$ (which covers our special situation for the category $\mathcal{O}$ ), the algebra $e A e$ still carries the structure of a left properly stratified algebra (induced from the quasi-hereditary structure on $A$ - actually, it would be enough to start with $A$ left properly stratified). The algebras occurring in the first part of the paper are all of this form. (See the remark on terminology in Section 7 for a comparison of the concept of left properly stratified algebras with other definitions of stratified algebras in the literature.)

A basic question for any abelian category occurring in Lie theory is to parameterize simple objects and to determine their characters or their multiplicities in other objects such as projective or standard objects. Since $e A e$ is left properly stratified and this structure is directly related to the quasi-hereditary structure of $A$, we get full answers to these questions (if the corresponding information on $A$ is available). In particular, characters of simples and their multiplicities in projective and standard objects are determined as consequences of Kazhdan-Lusztig conjecture (= theorem in our case).

We start Section 7 by recalling the definitions of quasi-hereditary and left properly stratified algebras and explaining the terminology and comparing it with similar notions used in the literature. Then we formulate and prove Theorem 5 , which is a sufficient criterion for an algebra $e A e$ to be standardly stratified if $A$ is quasihereditary (or, more generally, also standardly stratified). We also discuss multiplicity formulae and establish the existence of double centralizer properties (following the approach taken in [KSX]). In Section [8, we consider $\mathcal{S}$-subcategories in $\mathcal{O}$. Applying Theorem [5 to this setup yields Theorem [7, which reproves many results from [FKM1, FKM2] and [FKM3]. The assertions on double centralizer properties 
then reprove two of the main results of [FKM2, FKM3. Moreover, formulae for characters and multiplicities of simples are obtained.

Through all of the paper we restrict ourselves to considering modules in $\mathcal{O}$ which have integral support. This simplifies notation and arguments, but it is, in fact, not a restriction, since by Soergel's result $(\underline{\underline{S}})$ all the other blocks of $\mathcal{O}$ are equivalent to blocks having integral support (possibly for other Lie algebras); hence they share the same structure.

\section{Projectively PRESEnted Modules AND INJECTIVELY COPRESENTED MODULES}

This section fixes the abstract setup in which we will study Lie theoretic notions later on. In particular, it provides us with equivalences of categories which will be used. Most of the results are taken from Auslander's work [A], Sections 3 and 5. Modules always are left modules.

Let $A$ be a finite-dimensional associative algebra. By $\Lambda$ we denote the set of isomorphism classes of simple $A$-modules. The simple module $L(\lambda)$ has the projective cover $P(\lambda)$ and the injective envelope $I(\lambda)$.

Let $\Gamma$ be a subset of $\Lambda$. An $A$-module $M$ is $\Gamma$-projectively generated if it is a quotient of a direct sum of indecomposable projective modules indexed by elements of $\Gamma$ ( $\Gamma$-projectives $)$. It is $\Gamma$-projectively presented if it has a projective presentation $P_{2} \rightarrow P_{1} \rightarrow M \rightarrow 0$ by $\Gamma$-projectives. Dually, $M$ is $\Gamma$-injectively cogenerated if it is a submodule of a sum of indecomposable injective modules indexed by $\Gamma$ $(\Gamma$-injectives $)$, and it is $\Gamma$-injectively copresented if it has a copresentation by $\Gamma$ injectives.

Denote by $P(\Gamma)$ (resp. by $I(\Gamma)$ ) a direct sum of indecomposable projective (resp. injective) objects corresponding to the elements in $\Gamma$. Denote by $A_{\Gamma}$ the endomorphism ring of $P(\Gamma)$. The $\Gamma$-projectively presented modules can be characterized as follows in terms of the endomorphism ring $A_{\Gamma}$.

Proposition 1. For an A-module $M$ the following statements are equivalent:

(a) $M$ is $\Gamma$-projectively presented.

(b) The canonical morphism $P(\Gamma) \otimes_{A_{\Gamma}} \operatorname{Hom}_{A}(P(\Gamma), M) \rightarrow M$ is an isomorphism.

(c) There is an $A_{\Gamma}$-module $N$ such that the A-module $M$ is isomorphic to the induced module $P(\Gamma) \otimes_{A_{\Gamma}} N$.

Proof. See [A, 5.2].

The dual assertion reads as follows:

Proposition 2. For an A-module $M$ the following statements are equivalent:

(a) $M$ is $\Gamma$-injectively copresented.

(b) The canonical morphism $M \rightarrow \operatorname{Hom}_{A_{\Gamma}}\left(\operatorname{Hom}_{A}(P(\Gamma), A), \operatorname{Hom}_{A}(P(\Gamma), M)\right)$ is an isomorphism.

(c) There is an $A_{\Gamma}$-module $N$ such that the $A$-module $M$ is isomorphic to the coinduced module $\operatorname{Hom}_{A_{\Gamma}}\left(\operatorname{Hom}_{A}(P(\Gamma), A), N\right)$.

Proof. See [A, 5.5].

Up to a Morita equivalence, the endomorphism ring $A_{\Gamma}$ can be written as $e A e$ for some idempotent $e=e^{2} \in A$. Then the two canonical morphisms appearing above are the obvious morphisms $A e \otimes_{e A e} e M \rightarrow M$ and $M \rightarrow H_{e m e}(e A, e M)$. 
The functors occurring in the previous results actually yield equivalences of categories as follows.

Proposition 3. The full subcategory $\mathcal{B}(\Gamma)$ of $\Gamma$-projectively presented modules is equivalent to the category of $A_{\Gamma}$-modules, via induction and restriction. This gives $\mathcal{B}(\Gamma)$ an abelian structure. With respect to this abelian structure, the inclusion $\mathcal{B}(\Gamma) \subset A$-mod is right exact.

The full subcategory $\mathcal{C}(\Gamma)$ of $\Gamma$-injectively copresented modules is equivalent to the category of $A_{\Gamma}$-modules, via coinduction and restriction. This gives $\mathcal{C}(\Gamma)$ an abelian structure. With respect to this abelian structure, the inclusion $\mathcal{C}(\Gamma) \subset A$-mod is left exact.

In particular, $\mathcal{B}(\Gamma)$ and $\mathcal{C}(\Gamma)$ are equivalent categories.

Proof. This combines A, 5.1, 5.4 and 5.6].

Given any $A$-module, $M$, we can first map it to $A_{\Gamma^{-}}$-mod using the exact functor $\operatorname{Hom}_{A}(P(\Gamma), M)$ and then induce (resp. coinduce) it to a projectively presented module $M_{1}$ (resp. an injectively copresented module $M_{2}$ ). If $M$ is already $\Gamma$ generated, say $P_{1} \stackrel{f}{\rightarrow} M \rightarrow 0$, then $M_{1}$ is the sum of all cokernels of maps $P_{2} \rightarrow P_{1}$ with $P_{2} \in \operatorname{add}\left(P_{\Gamma}\right)$ which have image in the kernel of $f$. If $M$ is $\Gamma$-cogenerated, say $0 \rightarrow M \stackrel{g}{\rightarrow} I_{1}$, then $M_{2}$ is the intersection of all kernels of maps $I_{1} \rightarrow I_{2}$ with $I_{2} \in \operatorname{add}\left(I_{\Gamma}\right)$ which send $M$ to zero. Obviously, both maps, sending $M$ to $M_{1}$ or to $M_{2}$, are idempotent and functorial.

We fix the following notation: $M \mapsto \mathfrak{b}(M)=M_{1}$ and $M \mapsto \mathfrak{c}(M)=M_{2}$.

The object $\mathfrak{b}(M)$ together with the natural map $\mathfrak{b}(M) \rightarrow M$ is the left approximation (in the sense of $\mathrm{AR}$ ) of $M$ in the category of $\Gamma$-presented modules. The object $\mathfrak{c}(M)$ together with the natural map $M \rightarrow \mathfrak{c}(M)$ is the right approximation of $M$ in the category of $\Gamma$-copresented modules. Both approximations are unique, since the modules $M$ with $\mathrm{Hom}_{A}(P(\Gamma), M)=0$ form a localizing subcategory (see [A, Section 7]).

If a module $M$ is already $\Gamma$-generated, say $P \rightarrow M$, then $\mathfrak{b}(M)$ is the largest quotient of $P$ which maps onto $M$ and such that all the composition factors of the kernel of $\mathfrak{b}(M) \rightarrow M$ have indices not in $\Gamma$. That is, $\mathfrak{b}(M)$ is obtained from $M$ by maximal extension with non- $\Gamma$ composition factors.

If a module $M$ is already $\Gamma$-cogenerated, say $M \subset I$, then $\mathfrak{c}(M)$ is the largest submodule of $I$ which contains $M$ and such that all the composition factors of the quotient $\mathfrak{c}(M) / M$ have indices not in $\Gamma$. That is, $\mathfrak{c}(M)$ is obtained from $M$ by maximal coextension with non- $\Gamma$ composition factors.

\section{Complete modules in the sense of Enright}

Let $\mathfrak{A}=\mathfrak{N}_{-} \oplus \mathfrak{H} \oplus \mathfrak{N}_{+}$denote a semi-simple finite-dimensional complex Lie algebra with a fixed triangular decomposition, and let $\Delta=\Delta_{+} \cup \Delta_{-}$be the corresponding root system with basis $\pi$. Let $X_{\alpha}, \alpha \in \Delta, H_{\alpha}, \alpha \in \pi$ be a fixed Weyl-Chevalley basis of $\mathfrak{A}$. Let $\mathcal{O}$ denote the BGG-category of finitely generated, $\mathfrak{H}$-diagonalizable and locally $U\left(\mathfrak{N}_{+}\right)$-finite modules. For $\alpha \in \pi$ let $U_{\alpha}$ denote the Ore localization of $U(\mathfrak{A})$ with respect to $\left\{X_{-\alpha}^{m} \mid m \in \mathbb{N}\right\}$ (see $[\mathbf{M}]$ ). For $\alpha \in \pi$ we denote by $r_{\alpha}$ the composition of the following functors:

$$
U(\mathfrak{A})-\bmod \stackrel{U_{\alpha} \otimes_{U(\mathfrak{A})}-}{\longrightarrow} U_{\alpha}-\bmod \stackrel{\text { res }}{\longrightarrow} U(\mathfrak{A})-\bmod \stackrel{\text { locfin }}{\longrightarrow} U(\mathfrak{A})-\bmod ,
$$


where locfin is the functor of taking locally $X_{\alpha}$-finite part. We call this composition an elementary Enright completion (in Mathieu's version), see [De, $\mathrm{E}, \mathrm{M}]$. Clearly, $r_{\alpha}: \mathcal{O} \rightarrow \mathcal{O}$ is idempotent. A module $M \in \mathcal{O}$ is said to be complete in the sense of Enright, if $r_{\alpha}(M)=M$ for any $\alpha \in \pi$. Of course, there is a notion of relative completeness (or $\Gamma$-completeness) with respect to some set $\Gamma$ of simple roots. Obviously, the functor $r_{\alpha}$ is left exact, but, in general, not right exact. One also sees that $r_{\alpha}$ commutes with the parabolic induction. We also denote by $\mathfrak{A}^{\alpha}$ the Lie subalgebra of $\mathfrak{A}$ generated by $X_{ \pm \alpha}$. Clearly, $\mathfrak{A}^{\alpha} \simeq \mathfrak{s l}(2, \mathbb{C})$.

A module $M \in \mathcal{O}$ is said to have a quasi Verma flag, if there is a filtration, $M=M_{0} \supset M_{1} \supset \cdots \supset M_{n}=0$ such that each $M_{i} / M_{i+1}$ is a non-zero submodule of a Verma module (see [D] Chapter 7] for the definition and properties of Verma modules, which we denote by $M(\lambda)$ ).

Definition 1. The full subcategory $\mathcal{K}$ of $\mathcal{O}$ consists of all complete modules having a quasi Verma flag and integral support.

This was introduced in FKM3, where it was shown that $\mathcal{K}$ decomposes into a direct sum of module categories over local algebras. This will follow from our more general results later on. We note that in FKM3 the more complicated notation $s t \mathcal{F}_{\text {int }}$ was used instead of $\mathcal{K}$.

Our goal in this section is to show that a module (with integral support and from $\mathcal{O}$ ) is complete if and only if it is copresented by projective-injective modules. The first step deals with modules cogenerated by projective-injective modules:

Lemma 1. Let $M$ be an object in $\mathcal{O}$ with integral support. Then $M$ has a quasi Verma flag if and only if it is cogenerated by projective-injective modules.

Proof. The socle of a Verma module is the socle of a projective-injective module. Therefore, a module with a quasi Verma flag is cogenerated by projective-injective modules. Conversely, a projective module has a Verma flag, hence a quasi Verma flag. A submodule of a module with a quasi Verma flag itself must have a quasi Verma flag, which is obtained by restricting the original flag to the submodule.

We note that the length of a quasi Verma flag of a given module $M$ is an invariant of $M$, since it is equal to the number of composition factors of $M$ which are simple Verma modules.

The following lemma collects several assertions which were proved in FKM3. We repeat the easy proof for completeness.

Lemma 2. (a) Let $M$ have a quasi Verma flag. Then, for any $\alpha \in \pi$, the operator $X_{-\alpha}$ acts injectively on $M$. Hence $M \subset r_{\alpha}(M)$.

(b) Let $\lambda \in \mathfrak{H}^{*}$, let $\alpha \in \pi$ and let $s_{\alpha}$ be the reflection with respect to $\alpha$. Then $r_{\alpha}(M(\lambda)) \simeq M(\lambda)$ if $M(\lambda) \not \subset M\left(s_{\alpha}(\lambda)\right)$, and $r_{\alpha}(M(\lambda)) \simeq M\left(s_{\alpha}(\lambda)\right)$ if $M(\lambda) \subset M\left(s_{\alpha}(\lambda)\right)$.

(c) Let $M$ have a quasi Verma flag. Then $r_{\alpha}(M) / M$ is a direct sum of finitedimensional $\mathfrak{A}$-modules.

(d) Let $M$ and $N$ be two modules with quasi Verma flags. Suppose that $N \subset M$ and that both $M$ and $N$ are complete. Then any simple submodule of $M / N$ is a simple Verma module.

Proof. (a) follows from the fact that a Verma module is free over $U\left(\mathfrak{N}_{-}\right)$, hence torsion-free. 
(b) is a standard property (see $[\mathrm{E}, \mathrm{De}]$ ). Alternatively, it can be proven by using induction from a parabolic subalgebra. It is easy to produce a given Verma module from a Verma module over a smaller Lie algebra by such an induction. This induction functor commutes with completion, so the assertion follows from the corresponding $\mathfrak{s l}(2)$-statement, which can be easily verified by a direct calculation.

(c) also follows from an $\mathfrak{s l}(2)$-computation.

(d) Suppose that there is a simple submodule of $M / N$ which has the form $L(\lambda)$, for some integral $\lambda$, which does not belong to the closure of the antidominant Weyl chamber. Hence there is $\alpha \in \pi$ such that $L(\lambda)$ is a direct sum of finite-dimensional modules with respect to $\mathfrak{A}^{\alpha}$. Therefore, $M / N$ has elements on which $X_{-\alpha}$ acts in a locally nilpotent way. Since $r_{\alpha}$ is left exact, we have $r_{\alpha}(M / N) \supset r_{\alpha}(M) / r_{\alpha}(N)=$ $M / N \supset L(\lambda)$. This contradicts the fact that $X_{-\alpha}$ acts injectively on $r_{\alpha}(M / N)$ by the definition of $r_{\alpha}$.

Now we can restrict our attention to modules that are cogenerated by projectiveinjective modules. In fact:

Lemma 3. Let $M$ be a complete module with integral support. Then $M$ is cogenerated by projective-injective modules. In particular, $M$ has a quasi Verma flag.

Proof. Let $M \in \mathcal{O}$ be a module with integral support such that $r_{\alpha}(M)=M$ for any $\alpha \in \pi$. Then $r_{\alpha}(\operatorname{soc}(M)) \neq 0$ for all $\alpha \in \pi$; in particular, $X_{-\alpha}$ acts injectively on $\operatorname{soc}(M)$ for all $\alpha \in \pi$. In the integral case, the last statement means that $\operatorname{soc}(M)$ is a direct sum of simple Verma modules.

Now we are ready to state our result.

Theorem 1. Let $M \in \mathcal{O}$ be a module with integral support. Then $M$ is complete if and only if it is copresented by projective-injective modules.

Proof. Suppose that $M$ is complete. Then, by Lemma 3, it has a quasi Verma flag. Thus there is an inclusion $M \subset I$ for some projective-injective $I$. Using part (d) of Lemma 2 we get that $I / M$ has a socle which is a direct sum of simple Verma modules. Therefore, there exist a projective-injective module $I^{\prime}$ and a homomorphism $\varphi: I \rightarrow I^{\prime}$ such that $M$ equals the kernel of $\varphi$. It follows that $M$ is copresented by projective-injective modules.

Conversely, suppose that $M$ is copresented by projective-injective modules, say $M=\operatorname{ker}(\varphi)$ for some $\varphi: I \rightarrow I^{\prime}$ with $I$ and $I^{\prime}$ both projective-injective. In particular, the socle of the quotient $I / M$ is a direct sum of simple Verma modules. Part (c) of Lemma 2 implies that for any $\alpha$, the socle of the quotient $r_{\alpha}(M) / M$ (if non-zero) is a direct sum of simple modules which are not Verma modules. But $r_{\alpha}(M)$ is isomorphic to a submodule of $I$ as well; hence its image in $I / M$ must be zero. It follows that $r_{\alpha}(M)$ equals $M$.

Corollary 1. Each complete module from $\mathcal{O}$ has a quasi Verma flag. In particular, $\mathcal{K}$ is the subcategory of $\mathcal{O}$ consisting of all complete modules.

In Section 6, we will prove a stronger result.

\section{Relative theory AND the PARABolic CATEgory $\mathcal{O}$}

The results of the previous section generalize to statements on relative completions. Categories of relatively complete modules may look artificial at first. However, we show that they are equivalent to two other categories which have been 
studied before. These are, first, the parabolic category $\mathcal{O}(\mathcal{P}, \mathcal{K})$ which will be discussed in this section, and, second, a category of Harish-Chandra modules (see the next section).

In FKM3 the category $\mathcal{K}$ has been used to construct the following generalization of $\mathcal{O}$. Let $\mathfrak{G}$ be a semi-simple Lie algebra and let $\mathcal{P}$ be a parabolic subalgebra such that the semi-simple part of the Levi factor of $\mathcal{P}$ is isomorphic to $\mathfrak{A}$. Denote by $\mathcal{O}(\mathcal{P}, \mathcal{K})$ the full subcategory of the category $\mathcal{O}$ for $\mathfrak{G}$ (associated with the Borel subalgebra contained in $\mathcal{P}$ ) which consists of all modules possessing a decomposition into a direct sum of modules from $\mathcal{K}$, when viewed as $\mathfrak{A}$-modules. It has been shown in FKM3 that $\mathcal{O}(\mathcal{P}, \mathcal{K})$ has several nice properties. In particular, the blocks of this category possess a combinatorial description in the fashion of Soergel's description of the classical category $\mathcal{O}$. Now we can describe $\mathcal{O}(\mathcal{P}, \mathcal{K})$ in an abstract way, as we described $\mathcal{K}$ in the previous section.

Let $W$ denote the Weyl group of $\mathfrak{G}$ and $W_{\mathfrak{A}}$ the Weyl group of $\mathfrak{A}$. The longest representatives of the cosets $W / W_{\mathfrak{A}}$ will be called $\mathfrak{A}$-admissible elements. An injective (resp. projective, resp. semi-simple) module $I \in \mathcal{O}$ (resp. $P \in \mathcal{O}$, resp. $S \in \mathcal{O}$ ) will be called $\mathfrak{A}$-admissible provided all its indecomposable direct summands are injective envelopes of simple modules (resp. projective covers of simple modules, resp. simples) of the form $L(w(\lambda))$, where $w$ is $\mathfrak{A}$-admissible and $\lambda$ is dominant and $\mathfrak{A}$-integral.

If $W_{\mathfrak{A}}$ is generated by a simple reflection $s_{\alpha}$, then we will use the term $\alpha$ admissible as a synonym for $\mathfrak{A}$-admissible.

Lemma 4. Let $P$ (resp. I) be an $\mathfrak{A}$-admissible projective (resp. injective) module. Then the restriction of $P$ (resp. I) to $\mathfrak{A}$-modules is a direct sum of copies of projective-injective modules in the corresponding category $\mathcal{O}$.

Proof. By duality, it is enough to give the proof for $P$. By the parabolic analogue of the BGG-construction of projectives in $\mathcal{O}, P$ can be obtained from the projectiveinjective module $P^{\prime}$ over $\mathfrak{A}$ in two steps. First, induce it up to the parabolic subalgebra and then induce the result up to $\mathfrak{g}$. In both cases the result, as an $\mathfrak{A}$-module, decomposes into a direct sum $F \otimes P^{\prime}$, where $F$ is finite-dimensional. Hence $P$, as an $\mathfrak{A}$-module, is a direct sum of projective-injective modules.

Theorem 2. Let $M$ be in $\mathcal{O}$. Then the following statements are equivalent:

1. $M \in \mathcal{O}(\mathcal{P}, \mathcal{K})$.

2. $M$ is copresented by an $\mathfrak{A}$-admissible injective module.

3. $M$ has $\mathfrak{A}$-integral support and is relatively complete with respect to all simple roots of $\mathfrak{A}$.

Moreover, each relative completion functor coincides with the approximation functor associated with the category of modules copresented by $\mathfrak{A}$-admissible injective modules.

Proof. The equivalence of (1) and (3) follows directly from the definition and the remark that relative completions commute with parabolic induction.

The proof of the equivalence between (2) and (3) is based on the same arguments as the proof of Theorem 1 This is possible by Lemma 4, which permits us to copy the arguments using projective-injective modules. The description of completion in terms of injective copresentations also shows that the completion functor coincides with the approximation functor. 


\section{Summarizing ALL THE EQUivalenCeS}

Before we can state the result, we have to introduce another category by recalling some definitions and results from $\mathrm{BG}$.

Let $\theta$ be a central character. Consider $U(\mathfrak{G})$-bimodules which are algebraic (i.e., direct sums of finite-dimensional modules under the diagonal action of $\mathfrak{G}$ ) and which are, moreover, finitely generated as bimodules. By $\mathcal{H}_{f}^{1}(\theta)^{r}$ we denote the full subcategory of those modules on which the center acts through $\theta$ (here $f$ stands for finitely generated and $r$ for the right action of the center). Associated with $\theta$ is a dominant weight $\chi$. The stabilizer of $\chi$ defines a subalgebra $\mathfrak{A}$. Bernstein and Gelfand have shown ([BG, Theorem 5.9]) that a certain translation functor defines an equivalence between $\mathcal{H}_{f}^{1}(\theta)^{r}$ and the subcategory of $\mathcal{O}$ consisting of modules which are presented by $\mathfrak{A}$-admissible projective objects.

Summarizing all the equivalences constructed so far, we get

Corollary 2. The following five categories are equivalent:

1. The category of $\mathfrak{A}$-complete modules in $\mathcal{O}$ with integral support.

2. The integral part of the parabolic category $\mathcal{O}(\mathcal{P}, \mathcal{K})$.

3. The category $\mathcal{H}_{f}^{1}(\theta)^{r}$ of Harish-Chandra bimodules for integral singular $\theta$, whose stabilizing subalgebra is $\mathfrak{A}$.

4. The subcategory of $\mathcal{O}$ consisting of modules with integral support which are copresented by $\mathfrak{A}$-admissible injective modules.

The equivalences between (1), (2) and (4) above restrict canonically to equivalences between blocks. Each block is equivalent to the category eAe-mod for a suitable choice of $e$ and $A$.

Proof. The equivalence of (1), (2) and (4) is Theorem 2 The equivalence with (3) is the result of Bernstein and Gelfand combined with Proposition 3 . Finally, the statement about blocks is also contained in Proposition 3 .

We have to remark that, using the main result of [KM], one can add to the above list of five categories one more category, namely, the parabolic category $\mathcal{O}(\mathcal{P}, \mathcal{F})$, where $\mathcal{F}$ is generated by a simple generic Gelfand-Zetlin module. The precise definition of this category is quite long and technical, so we will not give it here. See [KM] for details.

One consequence of these equivalences is that it gives an abelian structure to all of these categories, which in the cases (1), (3) and (4) is not clear from the definitions. In particular, we get for free that each block of any of these categories has finitely many simple objects, each object has finite length, multiplicities of composition factors are well-defined, and so on.

Moreover, varying the defining parameters (i.e., the set of simple roots or the central character), we get different categories which one can compare, e.g., by using obvious functors between $e A e$-mod and $f A f$-mod, when $e$ and $f$ are the corresponding idempotents. In particular, the idea of comparing the corresponding completion functors will be developed in the next section.

\section{BRAID RELATIONS}

We keep the notation of the previous sections. Fix two simple roots $\alpha$ and $\beta$. Motivated by a problem posed by Enright $([\mathrm{E}])$, Bouaziz $([\mathrm{Bo}])$ and independently Deodhar $(\underline{\mathrm{De}})$, and later on also Joseph $([\overline{\mathrm{J}}])$, proved that completion functors 
satisfy braid relations. Joseph considered functors defined on all of $\mathcal{O}$, whereas Deodhar and Bouaziz restricted their attention to the subcategory of $\mathcal{O}$ which consists of all modules with integral support on which all $X_{-\gamma}, \gamma$ a positive root, act injectively. Joseph's completion functor is different from Mathieu's version which we are using. The two functors have different properties. Our functors do not satisfy braid relations on all of $\mathcal{O}$ (see the example at the end of this section), but they are idempotent functors. Joseph's functors do satisfy braid relations, but they are not idempotent.

Theorem 3 (Bouaziz, Deodhar). The braid relation $s_{\alpha} s_{\beta} \cdots=s_{\beta} s_{\alpha} \cdots$ in the Weyl group of $\mathfrak{A}$ implies the braid relation $r_{\alpha} r_{\beta} \cdots=r_{\beta} r_{\alpha} \cdots$ between compositions of completion functors on the subcategory $\mathcal{O}^{\alpha, \beta}$ of $\mathcal{O}$ which consists of all modules with integral support on which $X_{-\alpha}$ and $X_{-\beta}$ act injectively.

In this section we outline a short proof of this theorem which is based on our abstract framework.

Proof. We denote by $r_{l}$ (resp. $r_{r}$ ) the composition of functors occurring in the left (resp. right) hand side of the braid relation.

The first step is to reduce the problem to rank two situations. Let $\mathfrak{A}_{0}$ be the rank two subalgebra of $\mathfrak{A}$ corresponding to $\alpha$ and $\beta$. Denote its BGG-category by $\mathcal{O}^{\prime}$. The Lie theoretic definitions of completion show that the braid relations can be checked on the restriction of $\mathfrak{A}$-modules to $\mathfrak{A}_{0}$-modules. These restricted modules are direct sums of objects in $\mathcal{O}^{\prime}$. Denote by $\Gamma^{\prime}$ the index set of projective-injective objects in $\mathcal{O}^{\prime}$.

Lemma 5. Restricting from $U(\mathfrak{A})$ to $U\left(\mathfrak{A}_{0}\right)$ sends an injective object in $\mathcal{O}$ to a direct sum of injective objects in $\mathcal{O}^{\prime}$, and it sends a projective object in $\mathcal{O}$ to a direct sum of projective objects in $\mathcal{O}^{\prime}$.

Proof. Restriction preserves the defining properties of the category $\mathcal{O}$, except that the restriction of an object need not be finitely generated any more. By the PBWtheorem, induction from $U\left(\mathfrak{A}_{0}\right)$ to $U(\mathfrak{A})$ is exact. Therefore its right adjoint, restriction, sends injective objects in $\mathcal{O}$ to sums of injective objects in $\mathcal{O}^{\prime}$. Moreover, restriction and the operation of forming direct sums both commute with the dualities in these categories, which interchange projective and injective objects.

Another way to prove this statement is to first check the easy case of projective Verma modules. Then all other projectives can be obtained via exact tensoring with finite-dimensional modules.

In particular, projective-injective objects in $\mathcal{O}$ restrict to direct sums of projective-injective objects in $\mathcal{O}^{\prime}$. Moreover, if we denote by $\Gamma$ the index set of $\mathfrak{A}_{0}$-admissible injectives, then a $\Gamma$-cogenerated module $M$ in $\mathcal{O}$ restricts to a $\Gamma^{\prime}$ cogenerated module in $\mathcal{O}^{\prime}$, where in the latter case we allow infinite direct sums of projective-injective objects in the definition. It is enough to check the braid relations on finitely generated objects in $\mathcal{O}^{\prime}$.

Thus from now on we are working with $\mathfrak{A}_{0}$ only. We are given a module $M$ contained in a projective-injective module $I$. Applying $r_{\alpha}$ or $r_{\beta}$ to $M$ means extending $M$ inside $I$ by as many $\alpha$-non-admissible (or $\beta$-non-admissible) composition factors as possible. Let us call this an admissible extension of $M$.

Suppose for a moment that $M$ is a simple Verma module. It follows from part (b) of Lemma 2 that by applying either $r_{l}$ or $r_{r}$ to $M$ we always get the projective 
Verma module as a result. This implies that we can build any submodule of the projective Verma module, in particular any other Verma module, by successive admissible extensions of its socle (which is the given $M$ ).

Now we go back to the general situation:

Lemma 6. Given $M \subset I$, where $I$ is projective-injective, let $F$ be minimal with respect to inclusion such that it has the following properties: $F$ is a submodule of $I$; it contains $M$; and there exists a $\Delta$-filtration $F_{0} \subset F_{1} \subset \cdots \subset I$ of $I$ such that $F$ equals some $F_{i}$.

Then both $r_{l}(M)$ and $r_{r}(M)$ coincide with $F$.

Proof. Let $\Gamma$ be the indexing set of projective-injective modules. Then any Verma module has a socle of type $\Gamma$, i.e. any injective envelope of any simple occurring in the socle of a Verma module is projective. Therefore, there exists a map from $I$ into a direct sum of copies of $I$ which has kernel equal to $F$. Thus, $F$ is $\Gamma$-copresented and hence $\mathfrak{A}_{0}$-complete. It follows that $F$ contains both $r_{l}(M)$ and $r_{r}(M)$. By the minimality of $F$, all composition factors of type $\Gamma$ in $F$ are already in $M$. Hence, all the socles of the Verma subquotients of $F$ are in $M$. Thus, by the remarks preceding the statement of the lemma, both $r_{l}(M)$ and $r_{r}(M)$ contain $F$.

Now we see that both $r_{l}$ and $r_{r}$ applied to $M$ will give us the module $F$, which finishes the proof of the braid relations.

We recall that completion functors are idempotent: $r_{\alpha}=r_{\alpha}^{2}$. This is trivial, once they are identified with approximation functors.

We know already that completion functors are approximation functors, and so they must satisfy braid relations as well. More precisely, our proof shows that certain compositions of approximation functors are approximations with respect to intersections of the sets of injective modules involved. In fact, fix simple roots $\alpha$ and $\beta$ and suppose there is a braid relation $s_{\alpha} s_{\beta} \ldots=s_{\beta} s_{\alpha} \ldots$ in the Weyl group $W$. Denote by $\mathfrak{c}_{\alpha}$ the approximation functor with respect to the category of modules copresented by $\alpha$-admissible injectives, and by $\mathfrak{c}_{\beta}$ the approximation with respect to the category of modules copresented by $\beta$-admissible injectives. By $\mathfrak{c}_{\alpha, \beta}$ we denote the approximation with respect to the category of modules copresented by injectives which are both $\alpha$-admissible and $\beta$-admissible. In the above notation we immediately get the following.

Corollary 3. On the category of modules cogenerated by injectives which are both $\alpha$-admissible and $\beta$-admissible, the functors $\mathfrak{c}_{\alpha}$ and $\mathfrak{c}_{\beta}$ satisfy the same braid relation (as $s_{\alpha}$ and $s_{\beta}$ ). Moreover, the product in both sides of the relation coincides with $\mathfrak{c}_{\alpha, \beta}$, i.e., $\mathfrak{c}_{\alpha} \mathfrak{c}_{\beta} \cdots=\mathfrak{c}_{\alpha, \beta}=\mathfrak{c}_{\beta} \mathfrak{c}_{\alpha} \cdots$.

Applying the above arguments to $\mathfrak{A}$ instead of $\mathfrak{A}_{0}$ also shows:

Theorem 4. A complete module has a Verma flag.

This yields a lower bound for the representation type of the category of modules having a Verma flag.

To finish this section we present an example which explains why we have to restrict to the subcategory of $\Gamma$-cogenerated modules. In fact, on the full category $\mathcal{O}$, the completion functors $r_{\alpha}$ do not satisfy braid relations. Even worse, iterated compositions of such functors need not stabilize. This shows the difference between $r_{\alpha}$ and the original version of the completion functors, which, as it was shown by 
Joseph in [Jo], satisfy braid relations on the whole of $\mathcal{O}$, but are not idempotent. Only on the subcategory of $\Gamma$-cogenerated modules (where both completion functors coincide) does one have all these nice properties simultaneously.

Our example is the principal block of $\mathfrak{s l}(3)$. The projective Verma module has the following composition series:

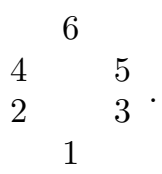

The indecomposable projective-injective module looks as follows:

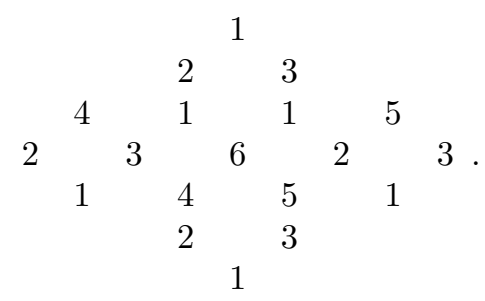

All other indecomposable projective modules are submodules of this one, and all other indecomposable injective modules are its quotients. We choose $\alpha$ in such a way that the completion functor $r_{\alpha}$ is the approximation with respect to the injective modules indexed by 1,3 and 5 . We choose $\beta$ corresponding to 1,2 and 4. Let $L$ be the simple module indexed by 2 . Under $r_{\alpha}$ it goes to zero. However, by $r_{\beta}$ it is sent to a module $M$ of length two, having simple socle 2 and simple top 5. Applying $r_{\alpha}$ now annihilates the socle, and hence the image coincides with the $r_{\alpha}$-image of the simple module indexed by 5 . The latter has length three and contains $L$ as a subquotient together with two other simples, indexed by 5 and 6 . The next step kills 5 and 6 , and we are back at the copy of $L$ we started with and to which we have to apply $r_{\alpha}$ again. Thus no braid $\left(r_{\alpha} r_{\beta}\right)^{n}$ ever will send $L$ to a complete module.

\section{QUASI-HEREDITARY ALGEBRAS AND LEFT PROPERLY STRATIFIED ALGEBRAS}

First we recall the definitions of quasi-hereditary algebras (CPS1] ) and of left properly stratified algebras (compare with [CPS2, APT, ADL, [KIM]). We fix an arbitrary ground field $k$. In this section, by an algebra we mean a finite-dimensional associative $k$-algebra.

Definition 2. Let $A$ be a finite-dimensional algebra, and $\Lambda$ the set of isomorphism classes of simple $A$-modules. Choose representatives $L(\lambda)$ of the elements of $\Lambda$. Let $\leq$ be a partial order on $I$. Then $(A, \leq)$ is called quasi-hereditary if and only if the following assertions are true:

(a) For each $\lambda \in \Lambda$, there exists a finite-dimensional $A$-module $\Delta(\lambda)$ with an epimorphism $\Delta(\lambda) \rightarrow L(\lambda)$ in such a way that the composition factors $L(\mu)$ of the kernel satisfy $\mu<\lambda$.

(b) For each $\lambda \in \Lambda$, the projective cover $P(\lambda)$ of $L(\lambda)$ maps onto $\Delta(\lambda)$ in such a way that the kernel has a finite filtration with factors $\Delta(\mu)$ satisfying $\mu>\lambda$. 
There exists an equivalent reformulation in terms of two-sided ideals. Call a twosided ideal $J$ of $A$ heredity if $J=A e A$ for a primitive idempotent $e$ and $\operatorname{End}_{A}(A e)$ is semi-simple. Then $A$ is quasi-hereditary if and only if it has a heredity chain $0 \subset J_{1} \subset J_{2} \subset \cdots \subset A$ of two-sided ideals, that is, for any $i$ the ideal $J_{i} / J_{i-1}$ is a heredity ideal in $A / J_{i-1}$ (CPS1]).

Definition 3. Let $A$ be a finite-dimensional algebra, and $\Lambda$ the set of isomorphism classes of simple $A$-modules. Choose representatives $L(\lambda)$ of the elements of $\Lambda$. Let $\leq$ be a partial order on $I$. Then $(A, \leq)$ is called left properly stratified if and only if the following assertions are true:

(c) For each $\lambda \in \Lambda$, there exists a finite-dimensional $A$-module $\Delta(\lambda)$ with an epimorphism $\Delta(\lambda) \rightarrow L(\lambda)$ in such a way that the composition factors $L(\mu)$ of the kernel satisfy $\mu \leq \lambda$.

(d) For each $\lambda \in \Lambda$, the projective cover $P(\lambda)$ of $L(\lambda)$ maps onto $\Delta(\lambda)$ in such a way that the kernel has a finite filtration with factors $\Delta(\mu)$ satisfying $\mu>\lambda$.

There also exists an equivalent definition in terms of two-sided ideals. A twosided ideal $J$ in $A$ is called left properly stratifying if $J$ is generated (as a two-sided ideal) by a primitive idempotent and $J$ is projective as a left $A$-module. Then the algebra $A$ is left properly stratified if and only if there exists an ordering $e_{1}, \ldots, e_{n}$ of the equivalence classes of primitive idempotents of $A$ such that for each $l$ the idempotent $e_{l}$ generates a left properly stratifying ideal in the quotient algebra $A /\left\langle e_{1}, \ldots, e_{l-1}\right\rangle$ (CPS2]). The corresponding chain of two-sided ideals is called a stratifying chain.

Remark on terminology. We are grateful to the referee for pointing out that the terminology used in the first version of this paper, and also in part of the literature, is misleading. In fact, there seem to be two definitions of standardly stratified algebras: the original one given in CPS2 and a more restrictive one which is used, for example, in ADL FKM1, FKM2, FKM3. In the general definition given in CPS2, one does not always use a complete set of representatives of all isoclasses of primitive idempotents in $A$. For example, in the sense of CPS2, any finitedimensional algebra $A$ has a standard stratification of length 1 with $A=J_{1}$ as the stratifying ideal. Another difference is that CPS2 uses the notion of quasi-partial order, and not that of partial order on the set of representatives of idempotents.

Our left properly stratified algebras are precisely the (left-handed version of the) standardly stratified algebras in the more restricted sense of [ADL, FKM1, [FKM2, FKM3. The name left properly stratified for our class of algebras was used, for example, in [KIM].

Left properly stratified algebras are, of course, standardly stratified in the sense of [CPS2]. In particular, in CPS2] it is shown that the derived category of a standardly stratified algebra admits a stratification, that is, in our case, a sequence of recollements by derived categories of local algebras, viz. the endomorphism rings $E_{l}$ of the modules $\Delta(\lambda)$. Any quasi-hereditary algebra is left properly stratified. A left properly stratified algebra $A$ is quasi-hereditary if and only if all the rings $E_{l}$ are simple, i.e., if and only if $A$ has finite global dimension ([CPS2, ADL]).

When $(A, \leq)$ is quasi-hereditary, there are two well-known ways of producing new quasi-hereditary algebras. If $I$ is an ideal in the partially ordered set $(\Lambda, \leq)$ (that is, $\lambda<\mu$ and $\mu \in I$ imply $\lambda \in I$ ) and $e$ is a complete sum of primitive 
idempotents representing elements of $I$, then $e A e$ is quasi-hereditary as well. If $I$ is a coideal, then $A / A e A$ is quasi-hereditary.

We are going to study algebras $e A e$ for a rather different choice of $e$, in order to cover the examples which we met in the first part of this paper. In the next section we will return to these examples.

From now on, $(A, \leq)$ is a fixed left properly stratified algebra with simple modules $L(\lambda)$ indexed by $\lambda \in \Lambda$. The projective cover of $L(\lambda)$ is denoted by $P(\lambda)$; the standard module mapping onto $L(\lambda)$ is $\Delta(\lambda)$. For any subset $I \subset \Lambda$, we denote by $e(I)$ a sum of pairwise orthogonal primitive idempotents $e(L)$ representing the elements $L \in \Lambda$. If $M$ is any module and $P$ is projective, then the trace of $P$ in $M$ is the sum of all homomorphic images of $P$ in $M$. If $M$ equals $A$ and $P$ is isomorphic to $A f$ for some idempotent $f$, then this trace coincides with the two-sided ideal AfA.

Theorem 5. Let $(A, \leq)$ be left properly stratified. Decompose the index set $\Lambda$ into a disjoint union $\Lambda=\bigcup_{i=1}^{n} \Lambda_{i}$ of subsets satisfying the following condition: If $\lambda \in \Lambda_{i}$ and $\mu \in \Lambda_{j}$ and $\lambda>\mu$, then $i<j$. Suppose moreover that each $\Lambda_{i}$ has a unique minimal element (with respect to the restriction of $\leq$ ), which we denote by $\lambda_{i}$. Let $e$ be a sum of corresponding idempotents $e\left(\lambda_{i}\right)$. Assume that the following two conditions are satisfied:

( $\dagger$ ) For all $i$ and $j$ and for all $\mu \in \Lambda_{j}$, there is an equality of filtration multiplicities $\left[P\left(\lambda_{i}\right): \Delta(\mu)\right]=\left[P\left(\lambda_{i}\right): \Delta\left(\lambda_{j}\right)\right] \cdot\left[P\left(\lambda_{j}\right): \Delta(\mu)\right]$.

( $\ddagger$ For all $i$ and $j$ and for all $\mu \in \Lambda_{j}$, in a standard filtration of the projective module $P\left(\lambda_{i}\right)$ all subquotients isomorphic to $\Delta(\mu)$ lie in the submodule generated by subquotients isomorphic to $\Delta\left(\lambda_{j}\right)$.

Then the algebra $(e A e, \leq)$ is left properly stratified.

Let us add two remarks. First, the submodule occurring in condition $(\ddagger)$ is well-defined because of the ordering conditions in the definition of left properly stratified algebras. Second, one can easily construct examples of quasi-hereditary algebras with a decomposition of $\Lambda$ such that either $(\dagger)$ or $(\ddagger)$ or both of them are not satisfied. Especially ( $\ddagger)$ is not easy to check in particular examples. However, in the case of the category $\mathcal{O}$ both conditions are not hard, as will be shown in the next section.

Proof. Let us first fix some notation. We fix a stratifying chain of $(A, \leq)$ as follows: $0=J_{0} \subset J_{1,1} \subset \cdots \subset J_{1, l_{1}} \subset J_{2,1} \subset \cdots \subset J_{2, l_{2}} \subset \cdots \subset A$, where $J_{1,1} \subset \cdots \subset J_{1, l_{1}}$ is the part corresponding to the indices in $\Lambda_{1}, J_{2,1} \subset \cdots \subset J_{2, l_{2}}$ corresponds to the indices in $\Lambda_{2}$, and so on. Multiplying by $e$ on both sides produces a chain of twosided ideals in $e A e$ which contains the following subchain: $0 \subset J_{1}=e J_{1, l_{1}} e \subset J_{2}=$ $e J_{2, l_{2}} e \subset \cdots \subset e A e$. By $\Delta(1,1), \ldots, \Delta\left(1, l_{1}\right)=\Delta\left(\lambda_{1}\right)$ we denote the $A$-standard modules corresponding to the indices up to $\lambda_{1}$.

We proceed by induction on $n$ (which counts the number of $\Lambda_{i}$ ). Assume that $(\dagger)$ and $(\ddagger)$ are satisfied. To begin the induction, we have to show that $J_{1}$ is a stratifying ideal.

Let us first show that $J_{1}$ is an idempotent ideal. By definition, $J_{1, l_{1}}$ is generated by an idempotent $f=e_{1,1}+\cdots+e_{1, l_{1}}$, that is, $J_{1, l_{1}}=A f A$. We have $e f=f e=e_{1, l_{1}}$. Thus $e_{1, l_{1}}$ is contained in $J_{1}$. We claim that $J_{1}$ is generated by $e_{1, l_{1}}$. As a left module, $J_{1, l_{1}}$ is the sum of the traces of the projective $A$-modules $A e_{1,1}, A e_{1,2}, \ldots, A e_{1, l_{1}}$ in $A$. Fix the projective module $A e_{i, l_{i}}$. For an index $j$ we 
denote by $T_{j}$ the trace of $A e_{1, j}$ in $A e_{i, l_{i}}$. We have to show that for each $j$, the space $e T_{j}$ is contained in $T_{l_{1}}$. Fix $j$. Since $A$ is left properly stratified, the trace $T_{j}$ as a left module is generated by the sum of all copies of $\Delta(1, j)$ in any standard filtration of $A e_{i, l_{i}}$. This filtration satisfies condition ( $\left.\ddagger\right)$, which implies that $e T_{j}$ is contained in a submodule generated by some copies of $\Delta\left(1, l_{1}\right)$, which in turn is contained in $T_{l_{1}}$, the trace of $A e_{1, l_{1}}$. Therefore, $e J_{1, l_{1}}$ equals $e A e_{1, l_{1}} A$, and multiplying by $e$ from the right implies that $e J_{1, l_{1}} e$ equals the trace of $A e_{1, l_{1}}$.

Next we have to show that $J_{1}$ is projective as a left $e A e$-module. We have just seen that it is the trace of the left projective $e A e$-module $e A e_{1, l_{1}}$. Fix a projective $A$-module $A_{i, l_{i}}$. We claim that the trace $T_{l_{1}}$ of $A e_{1, l_{1}}$ in $A_{i, l_{i}}$ is a direct sum of copies of $A e_{1, l_{1}}$. Once this has been shown, multiplying by $e$ on the left proves our claim. By definition, $T_{l_{1}}$ is a quotient of $\left(A e_{1, l_{1}}\right)^{m}$ for some $m \in \mathbb{N}$, which we choose minimal. In the previous paragraph we have shown that $T_{l_{1}}$ equals $A_{i, l_{i}} \cap J_{1, l_{1}}$. Thus $T_{l_{1}}$ has a filtration by standard modules. Moreover, we have already seen that all subquotients $\Delta(1, j)$ occurring in a standard filtration of $A_{i, l_{i}}$ are inside $M$. Condition ( $\dagger$ ) now tells us that the epimorphism $A e_{1, l_{1}}^{m} \rightarrow T_{l_{1}}$ must be injective as well, in order to get the correct filtration multiplicities.

The algebra $\left(A / J_{1, l_{1}}, \leq\right)$ satisfies the conditions $(\dagger)$ and $(\ddagger)$ as well. Thus we are done by induction.

The algebras $e A e$ which we have considered in the previous sections will be shown (in the next section) to be left properly stratified as a consequence of Theorem 5 For these algebras, it is of interest to parameterize simple modules and to describe their characters. For the algebras $A$ themselves this is known by the KazhdanLusztig conjecture (= theorem). We show now that such information is sufficient to solve the problem for $e A e$ as well. Keep the assumptions of Theorem 5 . Then the simple $e A e$-modules are parameterized by $\{1, \ldots, n\}$.

Proposition 4. Denote the eAe-standard modules by $\theta_{1}, \ldots, \theta_{n}$ and their projective covers by $Q_{1}, \ldots, Q_{n}$. Then the multiplicity $\left[\theta_{j}: e L\left(\lambda_{i}\right)\right]$ of the simple eAemodule eL $\left(\lambda_{i}\right)$ of index $i$ in $\theta_{j}$ is the sum of the multiplicities $\left[\Delta(\mu): L\left(\lambda_{i}\right)\right]$ of the simple A-module $L\left(\lambda_{i}\right)$ in A-standard modules indexed by elements in $\Lambda_{j}$ multiplied with their multiplicity $\left[P\left(\lambda_{j}\right): \Delta(\mu)\right]$ in the A-projective module $P\left(\lambda_{j}\right)$ :

$$
\left[\theta_{j}: e L\left(\lambda_{i}\right)\right]=\sum_{\mu \in \Lambda_{j}}\left[\Delta(\mu): L\left(\lambda_{i}\right)\right] \cdot\left[P\left(\lambda_{j}\right): \Delta(\mu)\right] .
$$

Moreover, for the multiplicities of standard modules in filtrations of projective modules, there is an equality: $\left[Q_{i}: \theta_{j}\right]=\left[P\left(\lambda_{i}\right): \Delta\left(\lambda_{j}\right)\right]$.

Proof. For any left properly stratified algebra, simple modules are parameterized by the indices of ideals in a stratifying chain of maximal length. Such a chain has been constructed in the proof of Theorem 5 .

To check the assertion on multiplicities, we proceed by induction on $n$. It is enough to look at the case $j=1$. Then the $e A e$-standard module $\theta_{1}$ is $e$ times the projective $A$-module $P\left(\lambda_{1}\right)$. Since $P\left(\lambda_{1}\right)$ is filtered by standard modules, the result follows.

Another basic property of the algebras arising in the context of category the $\mathcal{O}$ is a double centralizer property (generalizing Soergel's result [S]), which we consider now. 
Lemma 7. Let $A$ be an algebra, e some idempotent and $f$ a primitive idempotent contained in e (that is, ef $=f=f e$ ). If the projective $A$-module Af is injective as well and its top is isomorphic to its socle, then the same is true for the projective eAe-module eAf.

Proof. To say that $A f$ is an injective left $A$-module is equivalent to saying that its $k$-dual $\operatorname{Hom}_{k}(A f, k)$ is a projective right $A$-module. Multiplying by $e$ on both sides (and using $f=e f$ ) then yields that $H_{o m}(e A f, k)$ is a projective right $e A e-$ module. Hence $e A f$ is injective as a left $e A e$-module. If $L$ is the top of $A f$, then $e L$ is the top of $e A f$, which remains isomorphic to the socle.

Lemma 8. Let $A$ be an algebra, e some idempotent and $f$ a primitive idempotent contained in e. If Af is a faithful A-module, then eAf is a faithful eAe-module.

Proof. Clear.

Theorem 6. Let $A$ be an algebra, e some idempotent and $f$ a primitive idempotent contained in $e$. Suppose there is a resolution $0 \rightarrow A \rightarrow(A f)^{l} \rightarrow(A f)^{m}$ for some positive integers $l$ and $m$. Then there is a similar resolution over eAe. Moreover, there are two double centralizer properties:

$$
A \simeq \operatorname{End}\left(A f_{f A f}\right) \quad \text { and } \quad e A e \simeq \operatorname{End}\left(e A f_{f A f}\right) .
$$

Proof. The first statement is clear. Together with Lemma7, Lemma 8 and Proposition 2.6 in [KSX], this shows that the assumption of Theorem 2.8 (see also Theorem $2.10)$ in [KSX] is satisfied. This implies the double centralizer properties.

\section{The Algebras $e A e$ FOR BlOCKS OF $\mathcal{O}$}

We return to our previous setup: Let $\mathfrak{A}$ be a finite-dimensional simple Lie algebra over the complex numbers with a fixed triangular decomposition $\mathfrak{A}=\mathfrak{N}_{-} \oplus \mathfrak{H} \oplus$ $\mathfrak{N}_{+}$and let $\mathcal{O}$ be the BGG-category. Decompose $\mathcal{O}$ into blocks, and fix a block corresponding to an integral dominant weight $\lambda$. This block (like any other) is equivalent to $A$-mod for some quasi-hereditary algebra $(A, \leq)$. Denoting the Weyl group by $W$, the simple $A$-modules are parameterized by $\Lambda:=W \cdot \lambda$, the elements in the orbit of $\lambda$ under the dot action. The ordering $\leq$ is given by the Bruhat order on $W$. We also fix a subset of the basis of the root system and denote by $W_{0}$ the corresponding Weyl group. Suppose that $W_{0}$ contains the stabilizer subgroup of $W$ on $\lambda$. We partition $\Lambda=\bigcup_{i=1}^{n} \Lambda_{i}$ by cosets of $W_{0}$ in such a way that the condition in Theorem 5 is satisfied and each coset is ordered according to the Bruhat order on $W_{0}$ (to be more precise: on its cosets modulo the stabilizer of $\lambda$ ). In particular, $\Lambda_{1}$ is equal to the cosets of $W_{0}$ modulo the stabilizer of $\lambda$. Let $w_{0}$ be the unique longest element of $W_{0}$. The unique longest element $\lambda_{j}$ in $\Lambda_{j}$ is represented by $w_{0} w_{j}$ for a $w_{j} \in W$, which is the shortest element in the coset. Let $e$ be a sum of corresponding idempotents $e\left(\lambda_{i}\right)$.

Our approach in some sense is inverse to that of [FKM3. Here, we start with a quasi-hereditary algebra $A$ associated with a block of $\mathcal{O}$, pass to $e A e$ for the choice of $e$ as above, and prove properties of $e A e$ in this setup. Using the equivalences discussed above, we could go back to the Lie theoretic categories studied there and transport our results to these categories, thus reproving several results from [FKM2, [FKM3]. We refrain from explicitly stating all these consequences.

Theorem 7. With notation as above, the algebra $(e A e, \leq)$ is left properly stratified. 
Proof. We are going to check the conditions in Theorem 5. Throughout, we keep the above notation. We check condition $(\dagger)$ first.

Lemma 9. For all $i$ and $j$ and for all $\mu \in \Lambda_{j}$, there is an equality of filtration multiplicities $\left[P\left(\lambda_{i}\right): \Delta(\mu)\right]=\left[P\left(\lambda_{i}\right): \Delta\left(\lambda_{j}\right)\right] \cdot\left[P\left(\lambda_{j}\right): \Delta(\mu)\right]$. In particular, for all $i$ and $\mu \in \Lambda_{i}$ the multiplicity $\left[P\left(\lambda_{i}\right): \Delta(\mu)\right]$ equals one.

Proof. The proof proceeds by downwards induction along $W_{0}$. Induction starts with the trivial case $\lambda=\mu$. In the general case we apply BGG-reciprocity together with the well-known formula (see [J], 2.16) $\left[\Delta\left(w^{\prime} \cdot \lambda\right): L(w \cdot \lambda)\right]=\left[\Delta\left(w^{\prime} s \cdot \lambda\right): L(w \cdot \lambda)\right]$ (for $s$ a simple reflection and $w, w^{\prime}$ in $W_{0}$ such that $w^{\prime} s \cdot \lambda>w^{\prime} \cdot \lambda$ ) which transfers a multiplicity concerning $\mu$ to one concerning a shorter index. By the choice of $\Lambda_{i}$, one can go from $\lambda_{i}$ to any $\mu \in \Lambda_{i}$ in such a way that the assumptions of this formula are satisfied.

Before checking condition ( $\ddagger$ ) as well, we have to introduce some more notation. By $\mathfrak{A}_{0}$ we denote the semisimple Lie subalgebra of $\mathfrak{A}$ corresponding to $W_{0}$. By $U(\mathfrak{A})$ and $U\left(\mathfrak{A}_{0}\right)$ we denote the universal enveloping algebras of $\mathfrak{A}$ and $\mathfrak{A}_{0}$ respectively. By $\mathcal{O}^{\prime}$ we denote the BGG-category of $\mathfrak{A}_{0}$ defined by restricting the fixed triangular decomposition of $\mathfrak{A}$ to $\mathfrak{A}_{0}$.

Lemma 10. For all $i$ and $j$ and for all $\mu \in \Lambda_{j}$, in a standard filtration of the projective module $P\left(\lambda_{i}\right)$ all subquotients isomorphic to $\Delta(\mu)$ lie in a submodule generated by subquotients isomorphic to $\Delta\left(\lambda_{j}\right)$.

Proof. Recall that by Lemma 4 , the module $P\left(\lambda_{i}\right)$, as an $\mathfrak{A}_{0}$-module, is a direct sum of big projective modules. Now we proceed by induction on the number of $\mathfrak{A}_{0}$-slices of $P\left(\lambda_{i}\right)$ placed above $P_{\mathfrak{A}_{0}}\left(\lambda_{i}\right)$. We already know that the maximal level is a direct sum of big projectives over $\mathfrak{A}_{0}$, and these modules are generated by their simple tops. Inducing to $\mathfrak{A}$ gives that for $\mu \in \Lambda_{1}$ all $\Delta(\mu)$ belong to the submodule generated by $\Delta\left(\lambda_{1}\right)$. Moreover, because of the exactness of translation functors, the trace of $\Delta\left(\lambda_{1}\right)$ is a direct summand of $P\left(\lambda_{i}\right)$ as an $\mathfrak{A}_{0}$-module. Factoring out this direct summand, we complete our proof by induction.

Now we see that all conditions of Theorem 5 are satisfied, and the proof of Theorem 7 is complete.

In the case of $\mathcal{O}$, the formula of Proposition 4 simplifies to the following statement:

Corollary 4. Keep the assumptions of Theorem 7 and notation as in Proposi-

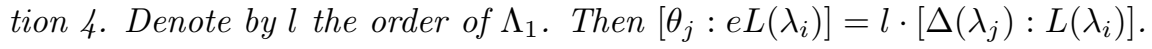

Proof. Combine Proposition 4 with Lemma 9 and with BGG-reciprocity.

Hence, composition and filtration multiplicities for $e A e$ are also given by the Kazhdan-Lusztig conjecture.

Applying the previous discussion of double centralizer properties, we get for free the following result. As remarked in [KSX], the methods of that paper also imply it directly.

Theorem 8. With notation as above, there is a double centralizer property

$$
e A e \simeq \operatorname{End}\left(e A f_{f A f}\right)
$$

where Af is the big projective module in $\mathcal{O}$. 
Proof. This is implied by Theorem [6] by using Theorem 3.2 in [KSX].

In $[S]$ it has been shown that $f A f$ is isomorphic to the coinvariant algebra, which in turn is isomorphic to the cohomology algebra of the corresponding flag variety. Therefore, our algebras eAe have a "combinatorial" description.

\section{ACKNOWLEDGEMENTS}

This research was done during the visit of the second author to Bielefeld University as an Alexander von Humboldt fellow. The financial support of the Humboldt Foundation and the hospitality of Bielefeld University are gratefully acknowledged. We would like to thank Wolfgang Soergel for pointing out the possibility of a connection between the categories studied in [FKM2 and those in BG. We thank Fred Van Oystaeyen for questions on [FKM3 which led us to develop the abstract approach of the present paper. We thank the referee for valuable comments and suggestions.

\section{REFERENCES}

[ADL] I.Agoston, V.Dlab and E.Lukacs, Stratified algebras. C. R. Math. Acad. Sci. Soc. R. Can. 20 (1998), no. 1, 22-28. MR 99h:16031

[A] M.Auslander, Representation theory of Artin algebras I. Commun. in Alg. 1 (1974), 177-268. MR 50:2240

[APT] M.Auslander, M.I.Platzeck and G.Todorov, Homological theory of idempotent ideals. Trans. Amer. Math. Soc. 332 (1992), 667-692. MR 92i:16008

[AR] M.Auslander and I.Reiten, Applications of contravariantly finite subcategories. Adv. Math. 86, 111-152 (1991). MR 92e:16009

[BG] I.N.Bernstein and S.I.Gelfand, Tensor products of finite- and infinite-dimensional representations of semisimple Lie algebras. Compositio Math. 41 (1980), 245-285. MR 82c: 17003

[BGG] I.N.Bernstein, I.M.Gelfand and S.I.Gelfand, A certain category of $\mathfrak{G}$-modules. (Russian). Funkcional. Anal. i Priložen. 10 (1976), no. 2, 1-8. MR 53:10880

[Bo] A.Bouaziz, Sur les représentations des algèbres de Lie semi-simples construites par T.Enright. In: Non-commutative harmonic analysis and Lie groups, Springer Lecture Notes in Mathematics 880 (1981), 57-68. MR 84j:17004

[BKM] T.Brüstle, S.König and V.Mazorchuk, The coinvariant algebra and representation types of blocks of category $\mathcal{O}$. Bull. London Math. Soc. 33 (2001), 669-681. CMP 2002:01

[CPS1] E.Cline, B.Parshall and L.Scott, Finite-dimensional algebras and highest weight categories. J. Reine Angew. Math. 391 (1988), 85-99. MR 90d:18005

[CPS2] E.Cline, B.Parshall and L.Scott, Stratifying endomorphism algebras. Mem. Amer. Math. Soc. 124 (1996), n. 591. MR 97h:16012

[De] V.V.Deodhar, On a construction of representations and a problem of Enright. Invent. Math. 57 (1980), 101-118. MR 81f:17004

[D] J.Dixmier, Enveloping algebras. Revised reprint of the 1977 translation. Graduate Studies in Mathematics, 11. American Mathematical Society, Providence, RI, 1996. MR 97c:17010

[DR] V.Dlab and C.M.Ringel, Every semiprimary ring is the endomorphism ring of a projective module over a quasihereditary ring. Proc. Amer. Math. Soc. 107 (1989), no. 1, 1-5. MR 89m:16033

[E] T.J.Enright, On the fundamental series of a real semisimple Lie algebra: their irreducibility, resolutions and multiplicity formulae. Annals Math., 110 (1979), 1-82. MR 81a:17003

[FKM1] V.Futorny, S.König and V.Mazorchuk, Categories of induced modules and standardly stratified algebras. To appear in Algebras and Representation Theory.

[FKM2] V.Futorny, S.König and V.Mazorchuk, A combinatorial description of blocks in $\mathcal{O}(\mathcal{P}, \Lambda)$ associated with $s l(2)$ induction. J. Algebra 231 (2000), no. 1, 86-103. MR 2001g:17008 
[FKM3] V.Futorny, S.König and V.Mazorchuk, $\mathcal{S}$-subcategories in $\mathcal{O}(\mathcal{P}, \Lambda)$. Manuscripta Math. 102 (2000), no. 4, 487-503. MR 2001h:17022

[J] J.C.Jantzen, Einhüllende Algebren halbeinfacher Lie-Algebren. Ergebnisse der Mathematik und ihrer Grenzgebiete (3), 3. Springer, 1983. MR 86c:17011

[Jo] A.Joseph, The Enright functor on the Bernstein-Gelfand-Gelfand category $\mathcal{O}$. Invent. Math. 67 (1982), 423-445. MR 84j:17005

[KlM] M.Klucznik and V.Mazorchuk, Parabolic decomposition for properly stratified algebras. Preprint 99-083, Bielefeld University. To appear in J. Pure Appl. Algebra. Available via www at "http://www.elsevier.nl/locate/jpaa/"

[KM] S.König and V.Mazorchuk, An equivalence of two categories of $\operatorname{sl}(n, \mathbb{C})$-modules. To appear in Algebras and Representation Theory.

[KSX] S.König, I.H.Slungård and C.C.Xi, Double centralizer properties, dominant dimension and tilting modules. J. Algebra 240 (2001), 393-412.

[M] O.Mathieu, Classification of irreducible weight modules. Ann. Inst. Fourier (Grenoble) 50 (2000), no. 2, 537-592. MR 2001h:17017

[R] C.M.Ringel, The category of modules with good filtrations over a quasi-hereditary algebra has almost split sequences. Math. Z. 208 (1991), no. 2, 209-223. MR 93c:16010

[S] W. Soergel, Kategorie $\mathcal{O}$, perverse Garben und Moduln über den Koinvarianten zur Weylgruppe. (in German) [Category $\mathcal{O}$, perverse sheaves and modules over the coinvariants for the Weyl group]. J. Amer. Math. Soc. 3 (1990), no. 2, 421-445. MR 91e:17007

Department of Mathematics and Computer Science, University of Leicester, UniverSITY ROAD, LEICESTER, LE1 7RH, ENGLAND

E-mail address: sck5@mcs.le.ac.uk

URL: http://www.mcs.le.ac.uk/ skoenig/

Department of Mathematics, Uppsala University, Box 480, SE-75106, Uppsala, SweDEN

E-mail address: mazor@math.uu.se

$U R L:$ http://www.math.uu.se/ mazor/ 